\title{
ON TWO QUANTUM VERSIONS OF THE DETAILED BALANCE CONDITION
}

\author{
FRANCO FAGNOLA \\ Dipartimento di Matematica, Politecnico di Milano \\ Piazza Leonardo da Vinci 32, I-20133 Milano, Italy \\ E-mail: franco.fagnola@polimi.it \\ VERONICA UMANITÀ \\ Dipartimento di Matematica, Università di Genova \\ Via Dodecaneso 35, I-16146 Genova, Italy \\ E-mail: veronica.umanita@fastwebnet.it
}

\begin{abstract}
Quantum detailed balance conditions are often formulated as relationships between the generator of a quantum Markov semigroup and the generator of a dual semigroup with respect to a certain scalar product defined by an invariant state. In this paper we survey some results describing the structure of norm continuous quantum Markov semigroups on $\mathcal{B}(\mathrm{h})$ satisfying a quantum detailed balance condition when the duality is defined by means of pre-scalar products on $\mathcal{B}(\mathrm{h})$ of the form $\langle x, y\rangle_{s}:=\operatorname{tr}\left(\rho^{1-s} x^{*} \rho^{s} y\right)(s \in[0,1])$ in order to compare the resulting quantum versions of the classical detailed balance condition. Moreover, we discuss the structure of generators of a quantum Markov semigroup which commute with the modular automorphism because this condition appears when we consider pre-scalar products with $s \neq 1 / 2$.
\end{abstract}

1. Introduction. Quantum detailed balance conditions generalise the well known notion of reversibility for classical Markov semigroups which is expressed by the symmetry in the $L^{2}$ space of an invariant measure.

The formulation for quantum open systems is not yet established and there are several versions involving different types of non-commutative symmetry and possibly also time reversal operations. The best known one for a norm continuous quantum Markov semigroup (QMS) $\mathcal{T}$ on the algebra $\mathcal{B}(\mathrm{h})$ of linear bounded operators on a complex separable Hilbert space h, with a faithful invariant normal state $\rho$ was introduced by Alicki [4, [5]

2000 Mathematics Subject Classification: 46L55, 47D05, 82B10, 82C10, 81S25.

Key words and phrases: quantum detailed balance, quantum Markov semigroup, Lindablad representation.

The paper is in final form and no version of it will be published elsewhere. 
(see also Frigerio, Gorini, Kossakowski, and Verri [16], [19]): a QMS $\mathcal{T}$ generated by $\mathcal{L}$ satisfies the quantum detailed balance condition if there exists a linear bounded operator $\widetilde{\mathcal{L}}$ on $\mathcal{B}(\mathrm{h})$ such that

$$
\begin{aligned}
& \operatorname{tr}(\rho x \mathcal{L}(y))=\operatorname{tr}(\rho \widetilde{\mathcal{L}}(x) y) \quad \text { for all } x, y \in \mathcal{B}(\mathrm{h}) \\
& \text { and } \quad \mathcal{L}-\widetilde{\mathcal{L}}=2 i[K, \cdot]
\end{aligned}
$$

for some self-adjoint operator $K \in \mathcal{B}(\mathrm{h})$.

Condition 11 means that $\widetilde{\mathcal{L}}$ is the dual operator of $\mathcal{L}$ with respect to the pre-scalar product $\langle x, y\rangle_{0}:=\operatorname{tr}\left(\rho x^{*} y\right)$ on $\mathcal{B}(\mathrm{h})$. The norm continuous semigroup $\widetilde{\mathcal{T}}=\left(\tilde{\mathcal{T}}_{t}\right)_{t>0}$ generated by $\widetilde{\mathcal{L}}$ is called the dual semigroup of $\mathcal{T}$ and satisfies the corresponding equation $\operatorname{tr}\left(\rho x \mathcal{T}_{t}(y)\right)=\operatorname{tr}\left(\rho \widetilde{\mathcal{T}}_{t}(x) y\right)$ for all $t \geq 0$. Since $\mathcal{L}$ is conditionally completely positive by condition (2), the quantum detailed balance condition implies that the dual semigroup $\widetilde{\mathcal{T}}$ of $\mathcal{T}$ is still a QMS. As a consequence, all the maps $\widetilde{\mathcal{T}}_{t}$ commute with the modular group $\left(\sigma_{t}\right)_{t \in \mathbb{R}}$ associated with $\rho$ (see Prop. 2.1 of [19]), $\sigma_{t}(a)=\rho^{i t} a \rho^{-i t}$; this is a quite restrictive condition. Indeed, Theorem 8 and the following remark show that it determines the structure of the generator of the QMS and quantum detailed balance follows under a simple additional condition.

Other pre-scalar products on the algebra, that are equivalent to the previous one in the commutative case, can be defined through the state $\rho$. The most natural are $\langle x, y\rangle_{s}:=\operatorname{tr}\left(\rho^{1-s} x^{*} \rho^{s} y\right)$ with $s \in[0,1]$, and we call $s$-dual semigroup of $\mathcal{T}$ a norm continuous semigroup $\widetilde{\mathcal{T}}^{(s)}$ satisfying $\operatorname{tr}\left(\rho^{1-s} \widetilde{\mathcal{T}}_{t}^{(s)}(x) \rho^{s} y\right)=\operatorname{tr}\left(\rho^{1-s} x \rho^{s} \mathcal{T}_{t}(y)\right)$ for all $t \geq 0$ and $x, y \in \mathcal{B}(\mathrm{h})$.

Clearly $\tilde{\mathcal{T}}^{(0)}=\tilde{\mathcal{T}}$. The other natural and popular choice is $s=1 / 2$ (see for example [3], 17] and [18]); the 1/2-dual semigroup $\mathcal{T}^{\prime}$ is called symmetric dual (or standard dual or $K M S$ dual) and is always a QMS i.e. the maps $\mathcal{T}_{t}^{\prime}$ are completely positive irrespectively of the commutation with $\left(\sigma_{t}\right)_{t>0}$. Another quantum detailed balance condition can be defined immediately by $(1)$ and 2 replacing $\widetilde{\mathcal{L}}$ by the generator $\mathcal{L}^{\prime}$ of the dual semigroup.

In this paper we compare the structure of generators of norm-continuous QMS on $\mathcal{B}(\mathrm{h})$ satisfying one of the above quantum versions of the classical detailed balance condition in order to understand which is the most natural and flexible. Moreover, we discuss the structure of generators of QMSs which commute with the modular group or, in an equivalent way, the modular automorphism $\sigma_{-i}$, because this condition appears as a consequence of the Alicki et al. quantum detailed balance condition.

Given a special GKSL representation of $\mathcal{L}$ of the form

$$
\mathcal{L}(x)=i[H, x]-\frac{1}{2} \sum_{k}\left(L_{k}^{*} L_{k} x-2 L_{k}^{*} x L_{k}+x L_{k}^{*} L_{k}\right)
$$

with $H=H^{*}, L_{k} \in \mathcal{B}(\mathrm{h})$ (see Section 2 for the precise definition), we first describe the structure of generators of QMSs whose dual semigroup is still a QMS: we find that the Hamiltonian $H$ is forced to commute with $\rho$, and the operators $L_{k}$ can be chosen as eigenvectors of the modular automorphism, i.e. $\rho L_{k} \rho^{-1}=\lambda_{k} L_{k}$ for some $\lambda_{k}>0$ (see Theorem 4. A special GKSL representation of $\mathcal{L}$ with these properties is called privileged. 
We stress the close relationship between the form of the $L_{k}$ 's and the structure of the spectrum of the modular automorphism (see Proposition 7). We also show the expression of the generator $\mathcal{L}$ in terms of the so-called ladder operators $\left(\left|e_{j}\right\rangle\left\langle e_{k}\right|\right)_{j, k}$, where $\left(e_{i}\right)_{i}$ is an orthonormal basis of eigenvectors of $\rho$ (see Theorem 8). This result was obtained before by Frigerio, Kossakowski, Gorini and Verri in [19] under the stronger assumption of quantum detailed balance.

A QMS commuting with $\left(\sigma_{t}\right)_{t \geq 0}$ satisfies the quantum detailed balance condition $\mathcal{L}-\widetilde{\mathcal{L}}=2 i[K, \cdot]$ if and only if $H-K=c$ for some real constant $c$ and there exists a unitary matrix $\left(u_{k l}\right)_{k l}$ such that $\lambda_{k}^{-1 / 2} L_{k}^{*}=\sum_{l} u_{k l} L_{l}$ (Theorem 14. The structure of the generator of a detailed balance QMS was already analyzed in [4] under the additional hypothesis that $\mathcal{L}$ and $\widetilde{\mathcal{L}}$ commute; here, we prove that this assumption is not necessary (see example 18). Moreover, we further characterize the generators of quantum detailed balance QMSs which commute with their dual map $\widetilde{\mathcal{L}}$ (Proposition 16), since in this case the generator $\mathcal{L}$ commutes with its derivation part $\delta_{H}(x)=[H, x]$ and it is possible to express the dual semigroup of $\mathcal{T}$ as $\widetilde{\mathcal{T}}_{t}(x)=\mathrm{e}^{-2 i t \delta_{H}}\left(\mathcal{T}_{t}(x)\right)=\mathcal{T}_{t}\left(\mathrm{e}^{-2 i t \delta_{H}}(x)\right)$.

We also characterize QMSs satisfying the "symmetric" (also called standard [10] or KMS [8, [18]) quantum detailed balance condition $\mathcal{L}-\mathcal{L}^{\prime}=2 i[K, \cdot]$, where $\mathcal{L}^{\prime}$ is the generator of $\mathcal{T}^{\prime}$ (Theorem 19): in particular, we show that this is equivalent to the symmetry of the completely positive part of $\mathcal{L}$, i.e. to the property

$$
\operatorname{tr}\left(\rho^{1 / 2} \Phi(x) \rho^{1 / 2} y\right)=\operatorname{tr}\left(\rho^{1 / 2} x \rho^{1 / 2} \Phi(y)\right),
$$

where $\Phi(x):=\sum_{k} L_{k}^{*} x L_{k}$ (see also [13] and [15]).

Although we do not find a definite answer the above results seem to indicate that the best known Alicki et al. quantum detailed balance condition is too restrictive and the "standard" or "symmetric" detailed balance condition is too weak because it involves only the completely positive part of the generator.

As a corollary of our results, we can also characterise (Theorem 21) generators of norm-continuous KMS-symmetric QMS, i.e. satisfying $\mathcal{L}=\mathcal{L}^{\prime}$, investigated by Accardi and Mohari [2, Goldstein and Lindsay [18], Cipriani [8], Park [23] and the references therein.

2. General setting. Let $\mathrm{h}$ be a complex separable Hilbert space and consider a norm continuous quantum Markov semigroup (QMS) $\mathcal{T}=\left(\mathcal{T}_{t}\right)_{t \geq 0}$ on $\mathcal{B}(\mathrm{h})$ with a faithful normal invariant state $\rho=\sum_{j \geq 1} \rho_{j}\left|e_{j}\right\rangle\left\langle e_{j}\right|$ with $\left(e_{j}\right)_{j \geq 1}$ an orthonormal basis of $\mathrm{h}$. We recall (Theorem 30.10 24]) that the generator $\mathcal{L}$ of $\mathcal{T}$ admits a special GKSL representation with respect to $\rho$ of the form (3) by means of operators $H=H^{*}$ and $\left(L_{k}\right)_{k \geq 1}$ in $\mathcal{B}(\mathrm{h})$ such that

(i) $\operatorname{tr}\left(\rho L_{k}\right)=0$ for each $k \geq 1$,

(ii) $\sum_{k \geq 1} L_{k}^{*} L_{k}$ is strongly convergent,

(iii) if $\sum_{k \geq 0}\left|c_{k}\right|^{2}<\infty$ and $c_{0}+\sum_{k \geq 1} c_{k} L_{k}=0$ for scalars $\left(c_{k}\right)_{k \geq 0}$ then $c_{k}=0$ for every $k \geq 0$.

Moreover, another family $\left\{H^{\prime}, L_{k}^{\prime}: k \geq 1\right\}$ of bounded operators in $\mathcal{B}(\mathrm{h})$ with $H^{\prime}$ selfadjoint, satisfies equation (3) and conditions (i) - (iii) if and only if the lengths of the 
sequences $\left(L_{k}\right)_{k \geq 1},\left(L_{k}^{\prime}\right)_{k \geq 1}$ are equal and

$$
H^{\prime}=H+\alpha, \quad L_{k}^{\prime}=\sum_{j} u_{k j} L_{j}
$$

for some scalar $\alpha$ and a unitary matrix $\left(u_{k j}\right)_{k j}$.

Introducing the bounded operator

on $\mathrm{h}$, we can also write $\mathcal{L}$ as

$$
G=-i H-\frac{1}{2} \sum_{k} L_{k}^{*} L_{k}
$$

$$
\mathcal{L}(a)=G^{*} a+\sum_{k} L_{k}^{*} a L_{k}+a G
$$

we say that such a representation is special if the operators $H=\left(G^{*}-G\right) / 2 i$ and $L_{k}$ give a special GKSL representation of $\mathcal{L}$. In this case, $G$ is unique up to a purely imaginary multiple of the identity operator. Indeed, if $G^{\prime},\left\{L_{k}^{\prime}\right\}$ give another special representation of $\mathcal{L}$, then $G^{\prime}=-i H^{\prime}-\frac{1}{2} \sum_{k} L_{k}^{\prime *} L_{k}^{\prime}$ fulfills

$$
G^{\prime}=-i H-i \alpha-\frac{1}{2} \sum_{k, j, m} \bar{u}_{k j} u_{k m} L_{j}^{*} L_{m}=-i H-i \alpha-\frac{1}{2} \sum_{j} L_{j}^{*} L_{j}=G-i \alpha
$$

for some $\alpha \in \mathbb{R}$, since the matrix $\left(u_{k j}\right)_{k j}$ is unitary.

Finally, let $\mathrm{k}$ be a Hilbert space with Hilbertian dimension equal to the length of the sequence $\left(L_{k}\right)_{k}$ (the multiplicity space of the completely positive part of the generator) and let $\left(f_{k}\right)$ be an orthonormal basis of $\mathrm{k}$. Defining a linear bounded operator $L: \mathrm{h} \rightarrow \mathrm{h} \otimes \mathrm{k}$ by $L u=\sum_{k} L_{k} u \otimes f_{k}$, any special GKSL representation of $\mathcal{L}$ can be expressed as (see Theorem 30.12 [24])

$$
\mathcal{L}(a)=i[H, a]-\frac{1}{2}\left(L^{*} L a-2 L^{*}\left(a \otimes \mathbb{1}_{\mathrm{k}}\right) L+a L^{*} L\right)
$$

for all $a \in \mathcal{B}(\mathrm{h})$, with $\operatorname{tr}\left(\rho L_{k}\right)=0$ for all $k$ and the set

(ii') $\left\{\left(a \otimes \mathbb{1}_{\mathrm{k}}\right) L u: a \in \mathcal{B}(\mathrm{h}), u \in \mathrm{h}\right\}$ total in $\mathrm{h} \otimes \mathrm{k}$.

If $H^{\prime}$ and $L^{\prime}: \mathrm{h} \rightarrow \mathrm{h} \otimes \mathrm{k}^{\prime}$ give another special representation of $\mathcal{L}$ with the above properties, then

$$
H^{\prime}=H+\alpha, \quad L^{\prime}=\left(\mathbb{1}_{\mathrm{h}} \otimes V\right) L
$$

for some $\alpha \in \mathbb{R}$ and $V: \mathrm{k} \rightarrow \mathrm{k}^{\prime}$ a unitary isomorphism.

Definition 1. The semigroup $\mathcal{T}$ is $s$-symmetric (with respect to $\rho$ ) if

$$
\operatorname{tr}\left(\rho^{1-s} \mathcal{T}_{t}(a) \rho^{s} b\right)=\operatorname{tr}\left(\rho^{1-s} a \rho^{s} \mathcal{T}_{t}(b)\right)
$$

for all $a, b \in \mathcal{B}(\mathrm{h})$ and $t \geq 0$.

We stress that for $s=1 / 2$ this is exactly the definition of KMS-symmetry with respect to the faithful state $\omega_{\rho}=\operatorname{tr}(\rho \cdot)$ (see for example [8], [18] and [23]). We refer to the lecture notes [9] for a discussion on this point. 
Definition 2. We say that $\mathcal{T}$ admits the $s$-dual semigroup with respect to $\rho$ if there exists a norm continuous semigroup $\widetilde{\mathcal{T}}^{(s)}=\left(\widetilde{\mathcal{T}}_{t}^{(s)}\right)_{t \geq 0}$ on $\mathcal{B}(\mathrm{h})$ such that

$$
\operatorname{tr}\left(\rho^{1-s} \widetilde{\mathcal{T}}_{t}^{(s)}(a) \rho^{s} b\right)=\operatorname{tr}\left(\rho^{1-s} a \rho^{s} \mathcal{T}_{t}(b)\right)
$$

for all $a, b \in \mathcal{B}(\mathrm{h})$ and $t \geq 0$.

When $s=0($ resp. $s=1 / 2)$ we denote $\widetilde{\mathcal{T}}^{(0)}$ (resp. $\tilde{\mathcal{T}}^{(1 / 2)}$ ) by $\tilde{\mathcal{T}}$ (resp. $\mathcal{T}^{\prime}$ ) and we call it the dual semigroup (resp. symmetric dual semigroup).

The $s$-dual semigroup $\widetilde{\mathcal{T}}^{(s)}$ is uniquely determined by condition (8); moreover, $\widetilde{\mathcal{T}}^{(s)}$ and its generator $\widetilde{\mathcal{L}}^{(s)}$ satisfy the following properties (see [13], Proposition 3.1 and 3.2):

1. $\rho^{1-s} \widetilde{\mathcal{T}}_{t}^{(s)}(a) \rho^{s}=\mathcal{T}_{* t}\left(\rho^{1-s} a \rho^{s}\right)$ for all $t \geq 0$, and $a, b \in \mathcal{B}(\mathrm{h})$;

2. $\widetilde{\mathcal{T}}_{t}^{(s)}(\mathbb{1})=\mathbb{1}$ and $\widetilde{\mathcal{T}}_{* t}^{(s)}(\rho)=\rho$ for all $t \geq 0$;

3. if $\widetilde{\mathcal{T}}_{t}^{(s)}$ is positive, then it is also normal;

4. $\rho^{1-s} \widetilde{\mathcal{L}}^{(s)}(a) \rho^{s}=\mathcal{L}_{*}\left(\rho^{1-s} a \rho^{s}\right)$ for all $a, b \in \mathcal{B}(\mathrm{h})$.

It is clear by 1 . that

$$
\widetilde{\mathcal{T}}_{t}^{(s)}(a)=\rho^{-(1-s)} \mathcal{T}_{* t}\left(\rho^{1-s} a \rho^{s}\right) \rho^{-s}
$$

on the dense subset $\rho^{s}(\mathrm{~h})=\rho(\mathrm{h})$ of $\mathrm{h}$; therefore, the symmetric dual semigroup (case $s=1 / 2$ ) is completely positive and then it is a QMS thanks to property 3 . However, if $s \neq 1 / 2$ the maps $\widetilde{\mathcal{T}}_{t}^{(s)}$ might not be positive and a fortiori not a QMS (see example 4.1 in 13 ).

Moreover, if $\mathrm{h}$ is finite dimensional, equation (9) defines a norm continuous semigroup of bounded operators on $\mathcal{B}(\mathrm{h})$ fulfilling relation (8), and so the $s$-dual semigroup exists. In the general case the situation is more difficult and it is not yet clear when $\mathcal{T}$ admits its dual semigroup: namely, we showed in [15] that there exists a QMS which satisfies equation (8) for $s=1 / 2$, but such a semigroup might not be norm continuous.

In the rest of paper, whenever we fix a value of $s$, we always assume that $\mathcal{T}$ admits its $s$-dual semigroup $\tilde{\mathcal{T}}^{(s)}$.

We now focus our attention on the cases $s=0$ and $s=1 / 2$. Indeed all the cases $s \neq 1 / 2$ have the same features, as shown in [13], Section 8 .

2.1. The quantum dual semigroup. In this section we analyze the duality for $s=0$ : by the general properties of $\widetilde{\mathcal{T}}^{(s)}$ we have

$$
\rho \widetilde{\mathcal{T}}_{t}(a)=\mathcal{T}_{* t}(\rho a) \quad \text { and } \quad \rho \widetilde{\mathcal{L}}(a)=\mathcal{L}_{*}(\rho a) .
$$

Since $\widetilde{\mathcal{T}}_{t}$ is not in general a positive map, we start finding those QMSs with dual which is still a QMS and describing the relationship between special GKSL representations of $\mathcal{L}$ and $\widetilde{\mathcal{L}}$.

Adapting an argument from Majewski and Streater (see [20], proof of Theorem 6), we can show (Theorem 3.1 of [13]) the following:

THEOREM 3. Let $a$ be the set of analytic elements for $\left(\sigma_{t}\right)_{t \in \mathbb{R}}$. The dual semigroup $\widetilde{\mathcal{T}}$ is a $Q M S$ if and only if one of the equivalent conditions holds:

(1) each $\mathcal{T}_{t}$ commutes with the modular automorphism $\sigma_{-i}$ on $a$;

(2) $\mathcal{L}$ commutes with $\sigma_{-i}$ on $a$. 
In terms of operators $H$ and $L_{k}$ which give a special representation of $\mathcal{L}$, we have the following result (see Theorem 4.3 of [13]).

THEOREM 4. The quantum dual semigroup $\widetilde{\mathcal{T}}$ is a $Q M S$ if and only if there exists a special GKSL representation of $\mathcal{L}$ by means of operators $H,\left(L_{k}\right)_{k}$ such that

(1) $\rho L_{k}=\lambda_{k} L_{k} \rho$ for some $\lambda_{k}>0$,

(2) $H$ commutes with $\rho$.

In particular, each of the operators $L_{k}^{*} L_{k}, G$ and $H$ commutes with $\rho$.

Definition 5. A special GKSL representation of $\mathcal{L}$ by means of operators $H,\left(L_{k}\right)_{k}$ is privileged if it satisfies conditions $(1),(2)$ of Theorem 4.

REMARK 6. Introducing the multiplicity space $\mathrm{k}$ of the completely positive part of $\mathcal{L}$, condition (1) of Theorem 4 is equivalent to

$$
\left(\rho \otimes \mathbb{1}_{\mathrm{k}}\right) L=\left(\mathbb{1}_{\mathrm{h}} \otimes D\right) L \rho,
$$

with $D=\operatorname{diag}\left(\lambda_{1}, \lambda_{2}, \ldots\right)=\sum_{k} \lambda_{k}\left|f_{k}\right\rangle\left\langle f_{k}\right|$, where $\left(f_{k}\right)_{k}$ is an orthonormal basis of $\mathrm{k}$.

Therefore, if $H^{\prime}, L_{k}^{\prime}$ give another privileged representation of $\mathcal{L}$, putting $L^{\prime}: \mathrm{h} \rightarrow \mathrm{h} \otimes \mathrm{k}$, $L^{\prime} u=\sum_{k} L_{k}^{\prime} u \otimes f_{k}$, and $D^{\prime}=\operatorname{diag}\left(\lambda_{1}^{\prime}, \lambda_{2}^{\prime}, \ldots\right)=\sum_{k} \lambda_{k}^{\prime}\left|f_{k}\right\rangle\left\langle f_{k}\right|$, we get

$$
H^{\prime}=H+\alpha, \quad L^{\prime}=\left(\mathbb{1}_{\mathrm{h}} \otimes V\right) L, \quad D^{\prime}=V D V^{*} .
$$

for some unitary operator $V$ on $\mathrm{k}$ and $\alpha \in \mathbb{R}$.

The identity $D^{\prime}=V D V^{*}$ means, roughly speaking, that $V$ is a change of coordinates that transforms $D$ into another diagonal matrix. Hence, when the $\lambda_{k}$ are all different, privileged GKSL representations of $\mathcal{L}$, if any, are unique up to a permutation of the operators $L_{k}$, a multiplication of each $L_{k}$ by a phase $\mathrm{e}^{i \theta_{k}}$ and a constant $\alpha$ in the Hamiltonian $H$. On the other hand, if some $\lambda_{k}$ 's are equal, then also unitary transformations of subspaces of $\mathrm{k}$ associated with the same $\lambda_{k}$ 's are allowed.

Condition (1) in Theorem 4 means that the operators $L_{k}$ are eigenvectors of the modular automorphism $\sigma_{-i}$; since $L_{k}=\sum_{i, j}\left\langle e_{i}, L_{k} e_{j}\right\rangle\left|e_{i}\right\rangle\left\langle e_{j}\right|$ and the so-called ladder operators $\left|e_{i}\right\rangle\left\langle e_{j}\right|$ are always eigenvectors of $\sigma_{-i}$ (with eigenvalues $\rho_{i} \rho_{j}^{-1}$ ), the form of the $L_{k}$ 's is then strictly connected with the structure of the spectrum of the modular automorphism.

Proposition 7. Suppose that $\mathcal{T}$ commutes with the modular automorphism and $\rho L_{k}=$ $\lambda L_{k} \rho$ for a fixed $\lambda>0$.

1. If there exists a unique pair $(i, j)$ such that $\rho_{i} \rho_{j}^{-1}=\lambda$, i.e. $\sigma_{-i}\left(\left|e_{i}\right\rangle\left\langle e_{j}\right|\right)=\lambda\left|e_{i}\right\rangle\left\langle e_{j}\right|$ we have $L_{k}=\alpha\left|e_{i}\right\rangle\left\langle e_{j}\right|$ for some $\alpha \in \mathbb{C}$.

2. If there exists $\left(i_{1}, j_{1}\right), \ldots,\left(i_{d}, j_{d}\right)$ such that $\sigma_{-i}\left(\left|e_{i_{l}}\right\rangle\left\langle e_{j_{l}}\right|\right)=\lambda\left|e_{i_{l}}\right\rangle\left\langle e_{j_{l}}\right|$ for $l=$ $1, \ldots, d$, then $L_{k}=\alpha_{1}\left|e_{i_{1}}\right\rangle\left\langle e_{j_{1}}\left|+\cdots+\alpha_{d}\right| e_{i_{d}}\right\rangle\left\langle e_{j_{d}}\right|$ for some $\alpha_{1}, \ldots, \alpha_{d} \in \mathbb{C}$.

Proof. Since $\rho=\sum_{l} \rho_{l}\left|e_{l}\right\rangle\left\langle e_{l}\right|$, the condition $\sigma_{-i}\left(\left|e_{i}\right\rangle\left\langle e_{j}\right|\right)=\lambda\left|e_{i}\right\rangle\left\langle e_{j}\right|$ is equivalent to $\rho_{i} \rho_{j}^{-1}=\lambda$. Moreover, by $\rho L_{k}=\lambda L_{k} \rho$ we have

$$
\rho_{l}\left\langle e_{l}, L_{k} e_{m}\right\rangle=\lambda \rho_{m}\left\langle e_{l}, L_{k} e_{m}\right\rangle
$$

for all $l, m$, so that $\left\langle e_{l}, L_{k} e_{m}\right\rangle \neq 0$ if and only if $\rho_{l} \rho_{m}^{-1}=\lambda$. 
It is worth noticing that, in case 1 , the self-adjoint operator $-\log (\rho)$ is generic in the sense of Accardi et al. (see e.g. [1]).

The generator of a QMS $\mathcal{T}$ commuting with $\sigma_{-i}$ can be expressed in terms of the ladder operators in the following way:

THEOREM 8. The QMS $\mathcal{T}$ commutes with the modular automorphism if and only if its generator assumes the form

$$
\mathcal{L}(x)=i[H, x]+\mathrm{uw}-\lim _{N} \sum_{r r^{\prime} s s^{\prime}=1}^{N} C_{r r^{\prime}} s s^{\prime}\left(P_{r r^{\prime}} x P_{s^{\prime} s}-\frac{1}{2}\left\{P_{r r^{\prime}} P_{s^{\prime} s}, x\right\}\right),
$$

where

(a) $P_{r r^{\prime}}=\left|e_{r}\right\rangle\left\langle e_{r^{\prime}}\right|$;

(b) $C_{r r^{\prime} s s^{\prime}}$ satisfies $\sum_{r r^{\prime} s s^{\prime}} \bar{\alpha}_{r r^{\prime}} C_{r r^{\prime} s s^{\prime}} \alpha_{s s^{\prime}} \geq 0$ for all sequences of scalars $\left(\alpha_{r r^{\prime}}\right)_{r r^{\prime}}$ for which the series converges;

(c) $\sum_{r r^{\prime} s s^{\prime}=1}^{N} C_{r r^{\prime} s s^{\prime}} P_{r r^{\prime}} P_{s s^{\prime}}$ is ultraweakly convergent as $N \rightarrow \infty$;

(d) $C_{r r^{\prime} s s^{\prime}} \rho_{r^{\prime}} \rho_{s}=C_{r r^{\prime}} s s^{\prime} \rho_{r} \rho_{s^{\prime}}$;

(e) $H=H^{*} \in \mathcal{B}(\mathrm{h})$ commutes with $\rho$.

Proof. Suppose that $\mathcal{L}$ commutes with the modular automorphism and let

$$
\mathcal{L}(x)=i[H, x]-\frac{1}{2}(\{\Phi(\mathbb{1}), x\}-2 \Phi(x))
$$

be a privileged representation of $\mathcal{L}$, with $\Phi(x)=\sum_{k} L_{k}^{*} x L_{k}$. Defining

$$
C_{r r^{\prime} s s^{\prime}}:=\operatorname{tr}\left(P_{s r} \Phi\left(P_{r^{\prime} s^{\prime}}\right)\right)=\sum_{k}\left\langle e_{r}, L_{k}^{*} e_{r^{\prime}}\right\rangle\left\langle e_{s^{\prime}}, L_{k} e_{s}\right\rangle<\infty,
$$

property (b) follows immediately for finite sequences. Then it can be extended to sequences of scalars $\left(\alpha_{r r^{\prime}}\right)_{r r^{\prime}}$ for which the series converges exploiting positivity of finite sums.

Since $\Phi$ commutes with the modular automorphism $\sigma_{-i}$ and ladder operator $P_{i j}$ are analytic, property $(\mathrm{d})$ follows from

$$
\begin{aligned}
C_{r r^{\prime} s s^{\prime}} & =\operatorname{tr}\left(P_{s r} \rho \Phi\left(\rho^{-1} P_{r^{\prime} s^{\prime}} \rho\right) \rho^{-1}\right)=\operatorname{tr}\left(\rho_{s}^{-1} P_{s r} \rho_{r} \Phi\left(\rho_{r^{\prime}}^{-1} P_{r^{\prime} s^{\prime}} \rho_{s^{\prime}}\right)\right) \\
& =C_{r r^{\prime}} s s^{\prime} \frac{\rho_{r}}{\rho_{r^{\prime}}} \frac{\rho_{s^{\prime}}}{\rho_{s}} .
\end{aligned}
$$

Set $Q_{N}=\sum_{r=1}^{N} P_{r r}, E_{N}(x)=Q_{N} x Q_{N}$ and

$$
\Phi_{N}(x):=E_{N} \Phi E_{N}(x)=\sum_{k} Q_{N} L_{k}^{*} Q_{N} x Q_{N} L_{k} Q_{N}=\sum_{r r^{\prime} s s^{\prime}=1}^{N} C_{r r^{\prime} s s^{\prime}} P_{r r^{\prime}} x P_{s^{\prime} s}
$$

for all $x \in \mathcal{B}(\mathrm{h})$, equation 12 is equivalent to

$$
\mathrm{uw}-\lim _{N}\left(\Phi_{N}(x)-\frac{1}{2}\left\{\Phi_{N}(\mathbb{1}), x\right\}\right)=\Phi(x)-\frac{1}{2}\{\Phi(\mathbb{1}), x\}
$$

for all $x \in \mathcal{B}(\mathrm{h})$.

Since $u w-\lim _{N} E_{N}(x)=x$ and $\Phi$ is normal, we get

$$
\operatorname{tr}\left(P_{i j}\left(\Phi_{N}(x)-\Phi(x)\right)\right)=\operatorname{tr}\left(P_{i j}\left(\Phi\left(E_{N}(x)\right)-\Phi(x)\right)\right) \rightarrow_{N} 0
$$


for all $i, j \in\{1, \ldots, N\}$, so that $\lim _{N} \operatorname{tr}\left(A\left(\Phi_{N}(x)-\Phi(x)\right)\right)=0$ for all $A$ in the linear span of the $P_{r s}$ 's; by the density of this set in the space of trace class operators on $\mathrm{h}$, we can conclude that uw- $\lim _{N} \Phi_{N}(x)=\Phi(x)$, due to the boundedness of the sequence $\left(\left|\operatorname{tr}\left(A\left(\Phi_{N}(x)-\Phi(x)\right)\right)\right|\right)_{N}$.

In particular, taking $x=\mathbb{1}$ we obtain property (c), and equation 13 follows then immediately.

Conversely, assume that $\mathcal{L}$ is written in the form $(12)$ and satisfies conditions (a)-(e). Thus we can write it as

$$
\mathcal{L}(x)=i[H, x]-\frac{1}{2}(\{\Phi(\mathbb{1}), x\}-2 \Phi(x))
$$

with $\Phi(x):=\mathrm{uw}-\lim _{N} \sum_{r r^{\prime} s s^{\prime}=1}^{N} C_{r r^{\prime} s s^{\prime}}\left(P_{r r^{\prime}} x P_{s^{\prime} s}\right)=\mathrm{uw}-\lim _{N} \Phi_{N}(x)$.

$\Phi$ is well defined and normal by (c); it is completely positive since any $\Phi_{N}$ is a completely positive map by (b). Therefore, $\Phi(x)=\sum_{k} L_{k}^{*} x L_{k}$ for some $L_{k} \in \mathcal{B}(\mathrm{h})$ such that $\sum_{k} L_{k}^{*} L_{k}$ is strongly convergent and $\mathcal{L}$ is represented in a GKSL form. To conclude we have to show that it commutes with the modular automorphism.

First of all, we note that

$$
\sum_{k}\left\langle e_{r}, L_{k}^{*} e_{r^{\prime}}\right\rangle\left\langle e_{s^{\prime}}, L_{k} e_{s}\right\rangle=\operatorname{tr}\left(P_{s r} \Phi\left(P_{r^{\prime} s^{\prime}}\right)\right)=\lim _{N} \operatorname{tr}\left(P_{s r} \Phi_{N}\left(P_{r^{\prime} s^{\prime}}\right)\right)=C_{r r^{\prime} s s^{\prime}} ;
$$

hence, by (d) follows $\operatorname{tr}\left(P_{s r} \Phi\left(P_{r^{\prime} s^{\prime}}\right)\right)=\operatorname{tr}\left(P_{s r} \rho \Phi\left(\rho^{-1} P_{r^{\prime} s^{\prime}} \rho\right) \rho^{-1}\right)$ for every $r, r^{\prime}, s, s^{\prime}$, which implies

$$
\operatorname{tr}\left(P_{s r} \Phi(x)\right)=\operatorname{tr}\left(P_{s r} \rho \Phi\left(\rho^{-1} x \rho\right) \rho^{-1}\right)
$$

for all $x \in a$ and $r, s$. This clearly means that $\Phi$ commutes with the modular automorphism, and so we can conclude by condition (e).

Remark 9. Notice that, according to [19] Theorem 2.2 p.99, the map $\mathcal{L}$ defined by (12) satisfies the quantum detailed balance condition in the sense of Alicki et al. (see Definition 12 with $s=0) C_{r r^{\prime} s s^{\prime}} \rho_{r}=C_{s^{\prime} s r^{\prime} r} \rho_{r^{\prime}}$ or, equivalently, $C_{r r^{\prime} s s^{\prime}} \rho_{s}=C_{s^{\prime} s r^{\prime} r} \rho_{s^{\prime}}$. These identities yield immediately $C_{r r^{\prime} s s^{\prime}} \rho_{r^{\prime}} \rho_{s}=C_{s^{\prime} s r^{\prime} r} \rho_{r^{\prime}} \rho_{s^{\prime}}=C_{r r^{\prime}} s s^{\prime} \rho_{r} \rho_{s^{\prime}}$.

We end this section establishing the relationship between the privileged GKSL representations of a generator $\mathcal{L}$ and a privileged representation of its 0-dual $\widetilde{\mathcal{L}}$ (see Proposition 4.3 and Theorem 4.4 in [13]).

THEOREM 10. If $\widetilde{\mathcal{T}}$ is a $Q M S$, then, for every privileged $G K S L$ representation of $\mathcal{L}$, by means of operators $H, L_{k}$, there exists a privileged $G K S L$ representation of $\widetilde{\mathcal{L}}$, by means of operators $\widetilde{H}, \widetilde{L}_{k}$ such that:

(1) $\widetilde{H}=-H-\alpha$ for some $\alpha \in \mathbb{R}$;

(2) $\widetilde{L}_{k}=\lambda_{k}^{-1 / 2} L_{k}^{*}$ for some $\lambda_{k}>0$.

As a consequence, $\widetilde{G}=G^{*}+i c$ for some $c \in \mathbb{R}$.

2.2. The symmetric dual semigroup. We now study the case $s=1 / 2$. We recall that the symmetric dual semigroup $\mathcal{T}^{\prime}$ of $\mathcal{T}$ is defined by

$$
\mathcal{T}_{t}^{\prime}(a)=\rho^{-1 / 2} \mathcal{T}_{* t}\left(\rho^{1 / 2} a \rho^{1 / 2}\right) \rho^{-1 / 2}
$$


for all $a \in \mathcal{B}(\mathrm{h})$. The name symmetric is justified by the left-right symmetry of multiplication by $\rho^{1 / 2}$ and $\rho^{-1 / 2}$. Equation 14 ensures that any map $\mathcal{T}_{t}^{\prime}$ is completely positive, contrary to the case $s=0$. Therefore the symmetric dual semigroup $\mathcal{T}^{\prime}$ is always a QMS with generator determined by

$$
\mathcal{L}^{\prime}(a)=\rho^{-1 / 2} \mathcal{L}_{*}\left(\rho^{1 / 2} a \rho^{1 / 2}\right) \rho^{-1 / 2} .
$$

It is well-known (see e.g. 8] or [13] Theorem 7.1 p. 358) that the symmetric dual $\mathcal{T}^{\prime}$ coincides with the 0 -dual $\widetilde{\mathcal{T}}$ if and only if each map $\mathcal{T}_{t}$ commutes with the modular automorphism $\sigma_{-i}$.

The relationship between the GKSL representation of generators $\mathcal{L}$ and $\mathcal{L}^{\prime}$ is the following.

THEOREM 11. For every special GKSL representation (5) of $\mathcal{L}$ there exists a special $G K S L$ representation of $\mathcal{L}^{\prime}$ by means of operators $G^{\prime}, L_{k}^{\prime}$ such that

(1) $G^{\prime} \rho^{1 / 2}=\rho^{1 / 2} G^{*}+i c \rho^{1 / 2}$ for some $c \in \mathbb{R}$,

(2) $L_{k}^{\prime} \rho^{1 / 2}=\rho^{1 / 2} L_{k}^{*}$.

Condition (2) ensures that the completely positive parts $\Phi$ and $\Phi^{\prime}$ of generators $\mathcal{L}$ and $\mathcal{L}^{\prime}$, respectively are in symmetric duality wrt $\rho$, i.e.

$$
\operatorname{tr}\left(\rho^{1 / 2} \Phi^{\prime}(a) \rho^{1 / 2} b\right)=\operatorname{tr}\left(\rho^{1 / 2} a \rho^{1 / 2} \Phi(b)\right)
$$

for all $a, b \in \mathcal{B}(\mathrm{h})$. In particular, this is equivalent to $\rho^{1 / 2} \Phi^{\prime}(a) \rho^{1 / 2}=\Phi_{*}\left(\rho^{1 / 2} a \rho^{1 / 2}\right), \Phi_{*}$ being the predual map of $\Phi$.

3. Quantum $s$-detailed balance condition. Considering the pre-scalar products $\langle\cdot, \cdot\rangle_{s}$ on $\mathcal{B}(\mathrm{h})$ the usual definition of quantum detailed balance introduced in [4], [5], [16] and [19], can be generalised as follows:

DEFINITION 12. A QMS $\mathcal{T}$ on $\mathcal{B}(\mathrm{h})$ satisfies the s-quantum detailed balance condition with respect to a normal faithful invariant state $\rho$, if its generator $\mathcal{L}$ and the generator $\widetilde{\mathcal{L}}^{(s)}$ of the $s$-dual semigroup $\tilde{\mathcal{T}}^{(s)}$ fulfill

$$
\mathcal{L}(a)-\widetilde{\mathcal{L}}^{(s)}(a)=2 i[K, a]
$$

for some bounded selfadjoint operator $K$ on $\mathrm{h}$ and for all $a \in \mathcal{B}(\mathrm{h})$.

Clearly the identity 16 implies that $\widetilde{\mathcal{L}}^{(s)}$ is conditionally completely positive, so that the $s$-dual semigroup of a QMS satisfying the $s$-detailed balance condition is always a QMS. Moreover the selfadjoint operator $K$ commutes with $\rho$, since $\rho$ is an invariant state for both $\mathcal{T}$ and $\widetilde{\mathcal{T}}^{(s)}$.

The $s$-quantum detailed balance condition is equivalent to $\mathcal{L}-i[K, \cdot]=\widetilde{\mathcal{L}}^{(s)}+i[K, \cdot]$ for some selfadjoint operator $K$; therefore, by comparing the special GKSL representations of $\mathcal{L}-i[K, \cdot]$ and $\widetilde{\mathcal{L}}^{(s)}+i[K, \cdot]$ we find

$$
G-i K=\widetilde{G}^{(s)}+i K+i c, \quad \widetilde{L}_{k}^{(s)}=\sum_{j} u_{k j} L_{j}
$$

for some unitary matrix $\left(u_{k j}\right)_{k j}$ and some $c \in \mathbb{R}$. 
Hence, a first characterization of the $s$-quantum detailed balance condition is the following:

THEOREM 13. $\mathcal{T}$ satisfies the quantum s-detailed balance condition $\mathcal{L}-\widetilde{\mathcal{L}}^{(s)}=2 i[K, \cdot]$ if and only if the equivalent facts below hold:

(i) for all special GKSL representations of generators $\mathcal{L}$ and $\widetilde{\mathcal{L}}^{(s)}$ by means of operators $G, L_{k}$ and $\widetilde{G}^{(s)}, \widetilde{L}_{k}^{(s)}$ respectively, we have

(1) $\widetilde{G}^{(s)}=G-i(2 K+c)$ for some $c \in \mathbb{R}$,

(2) $\widetilde{L}_{k}^{(s)}=\sum_{j} u_{k j} L_{j}$ for some unitary matrix $\left(u_{k j}\right)_{k j}$;

(ii) for all special GKSL representations of generators $\mathcal{L}$ and $\widetilde{\mathcal{L}}^{(s)}$ by means of operators $H, L_{k}$ and $\widetilde{H}^{(s)}, \widetilde{L}_{k}^{(s)}$ respectively, we have

$\left(1^{\prime}\right) \widetilde{H}^{(s)}=H-(2 K+c)$ for some $c \in \mathbb{R}$,

$\left(2^{\prime}\right) \widetilde{L}_{k}^{(s)}=\sum_{j} u_{k j} L_{j}$ for some unitary matrix $\left(u_{k j}\right)_{k j}$.

In the following we will specialize this result to the cases $s=0$ and $s=1 / 2$.

3.1. Quantum detailed balance condition. Assume now $s=0$; in this case, we speak of quantum detailed balance condition.

Comparing Theorems 13 and 10 , we can characterize the generator of a QMS satisfying the quantum detailed balance condition as follows:

THEOREM 14. The following conditions are equivalent:

(i) $\mathcal{T}$ satisfies the detailed balance condition $\mathcal{L}-\widetilde{\mathcal{L}}=2 i[K, \cdot]$;

(ii) there exists a privileged GKSL representation of $\mathcal{L}$, by means of operators $H, L_{k}$, such that

(1) $H=K+c$ for some $c \in \mathbb{R}$,

(2) $\lambda_{k}^{-1 / 2} L_{k}^{*}=\sum_{j} u_{k j} L_{j}$ for some $\lambda_{k}>0$ and some unitary operator $U=\left(u_{k j}\right)_{k j}$ on $\mathrm{k}$;

(iii) every privileged GKSL representation of $\mathcal{L}$ is given by operators $H, L_{k}$ satisfying conditions (1), (2).

In particular, the unitary matrix $U$ satisfies $\bar{U}=U^{*}$.

Proof. For the equivalence of items (i)-(iii) see the proof of Theorem 5.1 in [13]. We show now only the last statement.

Since $\lambda_{j}^{-1 / 2} L_{j}=\sum_{i} \bar{u}_{j i} L_{i}^{*}$ by condition (2) in (ii), substituting in $\lambda_{k}^{-1 / 2} L_{k}^{*}=\sum_{j} u_{k j} L_{j}$ we have

$$
\lambda_{k}^{-1 / 2} L_{k}^{*}=\sum_{i j} u_{k j} \bar{u}_{j i} \lambda_{j}^{1 / 2} L_{i}^{*},
$$

so that $\lambda_{k}^{-1 / 2} \delta_{k i}=\sum_{j} \lambda_{j}^{1 / 2} u_{k j} \bar{u}_{j i}$ from the linear independence of the $L_{k}$. This clearly means

$$
D^{-1 / 2}=U D^{1 / 2} \bar{U}
$$


where $D=\operatorname{diag}\left(\lambda_{1}, \lambda_{2}, \ldots\right)$. Moreover, set $L_{k}^{\prime}:=\lambda_{k}^{-1 / 2} L_{k}^{*}$, equation (2) in (ii) implies that the GKSL representation given by $H, L_{k}^{\prime}$ is also privileged and $\rho L_{k}^{\prime}=\lambda_{k}^{-1} L_{k}^{\prime} \rho$, that is $\lambda_{k}^{\prime}=\lambda_{k}^{-1}$. Therefore, by Remark 6 we get

$$
D^{-1}=U D U^{*}
$$

so that $D^{-1 / 2}=U D^{1 / 2} U^{*}$ by spectral calculus, since $U$ is unitary.

The conclusion follows by substituting in equation (17).

REMARK 15. As a consequence of the previous Theorem, if $\mathcal{T}$ satisfies the quantum detailed balance condition, the completely positive part $\Phi(x)=\sum_{k} L_{k}^{*} x L_{k}$ in a privileged representation of $\mathcal{L}$ is symmetric wrt $\rho$. Indeed,

$$
\begin{aligned}
\operatorname{tr}(\rho \Phi(x) y) & =\sum_{k} \operatorname{tr}\left(\lambda_{k}^{-1} L_{k}^{*} \rho x L_{k} y\right) \\
& =\sum_{k} \operatorname{tr}\left(\rho x\left(\lambda_{k}^{-1 / 2} L_{k}\right) y\left(\lambda_{k}^{-1 / 2} L_{k}^{*}\right)\right)=\operatorname{tr}(\rho x \Phi(y)),
\end{aligned}
$$

since $\lambda_{k}^{-1 / 2} L_{k}^{*}=\sum_{j} u_{k j} L_{j}$ (and so $\lambda_{k}^{-1 / 2} L_{k}=\sum_{j} \bar{u}_{k j} L_{j}^{*}$ ) with $\left(u_{k j}\right)_{k j}$ a unitary matrix.

Theorem 14 generalizes a result of Alicki ([4], Theorem 3), in which he characterized (in the finite-dimensional case) generators $\mathcal{L}$ satisfying the quantum detailed balance condition under the additional hypothesis that the operator $\mathcal{L}$ is normal, i.e. it commutes with $\widetilde{\mathcal{L}}$. This assumption implies that $\mathcal{T}$ commutes with the modular automorphism (see Lemma 3 of [4]), but it is not necessary, as shown by Example 18.

Indeed, note first that

Proposition 16. Assume $\mathcal{T}$ satisfies the quantum detailed balance condition and consider a privileged $G K S L$ representation of $\mathcal{L}$ by means of operators $H,\left(L_{k}\right)_{k}$. Set $\delta_{H}=$ $[H, \cdot], \mathcal{L}_{0}=\mathcal{L}-i \delta_{H}$ and $\Phi(x)=\sum_{k} L_{k}^{*} x L_{k}$, the statements below are equivalent:

(i) $\mathcal{L} \widetilde{\mathcal{L}}=\widetilde{\mathcal{L}} \mathcal{L}$;

(ii) $\mathcal{L}_{0} \delta_{H}=\delta_{H} \mathcal{L}_{0}$;

(iii) $\Phi \delta_{H}=\delta_{H} \Phi$.

Proof. By Theorem 14 we have $\widetilde{\mathcal{L}}=\mathcal{L}-2 i \delta_{H}$, so that

$$
\begin{aligned}
& \mathcal{L} \widetilde{\mathcal{L}}(x)=\mathcal{L}^{2}(x)-2 i \mathcal{L} \delta_{H}(x)=\mathcal{L}^{2}(x)-2 i \mathcal{L}_{0} \delta_{H}(x)+2 \delta_{H}^{2}(x) \\
& \widetilde{\mathcal{L}} \mathcal{L}(x)=\mathcal{L}^{2}(x)-2 i \delta_{H} \mathcal{L}(x)=\mathcal{L}^{2}(x)-2 i \delta_{H} \mathcal{L}_{0}(x)+2 \delta_{H}^{2}(x) .
\end{aligned}
$$

The equivalence (i) $\Leftrightarrow$ (ii) is now self-evident.

To conclude it is enough to note that $\mathcal{L}_{0}(x)=\Phi(x)-\frac{1}{2}\{\Phi(\mathbb{1}), x\}$ and $\Phi(\mathbb{1})=\sum_{k} L_{k}^{*} L_{k}$ commutes with $H$ by Theorem 4 .

REMARK 17. The previous proposition allows us to know when the quantum detailed balance condition can be expressed in a simple way in terms of $\mathcal{T}$ and its dual $\tilde{\mathcal{T}}$; indeed, when $\mathcal{L}_{0}$ and the derivation $\delta_{H}$ commute, the property $\mathcal{L}-\widetilde{\mathcal{L}}=2 i[H, \cdot]$ is equivalent to

$$
\widetilde{\mathcal{T}}_{t}=e^{t \widetilde{\mathcal{L}}}=e^{t \mathcal{L}} e^{-2 i \delta_{H}}=\mathcal{T}_{t} e^{-2 i \delta_{H}} .
$$

Unfortunately, the following example shows that such a commutation is not, in general, a consequence of the quantum detailed balance condition. 
ExAmple 18. Let $\mathrm{h}=\mathbb{C}^{3}$ with canonical basis $\left(\left|e_{j}\right\rangle\right)_{j=1}^{3}$ and let $D$ be the operator $z\left|e_{1}\right\rangle\left\langle e_{2}|+w| e_{2}\right\rangle\left\langle e_{3}\right|$ on h with $z, w \in \mathbb{C}-\{0\}$. Put

$$
L_{1}=D, \quad \text { and } \quad L_{2}=\lambda D^{*}
$$

for some $\lambda>0, \lambda \neq 1$, and consider the conditionally completely positive map $\mathcal{L}_{0}$ on $\mathcal{B}\left(\mathbb{C}^{3}\right)$ defined as in 3 with $H=0$. A straightforward computation shows that a faithful normal state $\rho=\sum_{j=1}^{3} \rho_{j}|j\rangle\langle j|$ satisfies $\mathcal{L}_{0 *}(\rho)=0$ if and only if

$$
\lambda^{2}=\rho_{2} / \rho_{1}=\rho_{3} / \rho_{2},
$$

i.e., by the normalisation $\rho_{1}+\rho_{2}+\rho_{3}=1$,

$$
\rho_{j}=\lambda^{2(j-1)} /\left(1+\lambda^{2}+\lambda^{4}\right), \quad \text { for } \quad j=1,2,3 .
$$

The operator $D$ satisfies $\rho D=\lambda^{-2} D \rho$.

Consider the generator $\mathcal{L}$ of a QMS defined by

$$
\mathcal{L}(x)=i[\rho, x]-\frac{1}{2} \sum_{k=1}^{2}\left(L_{k}^{*} L_{k} x-2 L_{k}^{*} x L_{k}+x L_{k}^{*} L_{k}\right)
$$

that clearly admits $\rho$ as a faithful invariant state. Moreover, since $\rho L_{1}=\lambda^{-2} L_{1} \rho$ and $\rho L_{2}=\lambda^{2} L_{2} \rho$, with our notation we have $\lambda_{1}=\lambda^{-2}$ and $\lambda_{2}=\lambda^{2}$. Therefore equation 20 defines a privileged GKSL representation of $\mathcal{L}$ with $H=\rho$, and the quantum detailed balance condition is fulfilled because

$$
\left(\begin{array}{c}
\lambda_{1}^{-1 / 2} L_{1}^{*} \\
\lambda_{2}^{-1 / 2} L_{2}^{*}
\end{array}\right)=\left(\begin{array}{c}
\lambda D^{*} \\
D
\end{array}\right)=\left(\begin{array}{c}
L_{2} \\
L_{1}
\end{array}\right)=\left(\begin{array}{ll}
0 & 1 \\
1 & 0
\end{array}\right)\left(\begin{array}{c}
L_{1} \\
L_{2}
\end{array}\right)
$$

We finally show that $\mathcal{L}$ is not normal, i.e. by Proposition 16 , that the completely positive part $\Phi(x)=\sum_{k=1}^{2} L_{k}^{*} x L_{k}$ does not commute with $\delta_{H}$. We start computing

$$
\begin{aligned}
\Phi \delta_{H}(x) & =D^{*} \rho x D-D^{*} x \rho D+\lambda^{2} D \rho x D^{*}-\lambda^{2} D x \rho D^{*}, \\
\delta_{H} \Phi(x) & =\rho D^{*} x D-D^{*} x D \rho+\lambda^{2} \rho D x D^{*}-\lambda^{2} D x D^{*} \rho \\
& =\lambda^{2} D^{*} \rho x D-\lambda^{2} D^{*} x \rho D+D \rho x D^{*}-D x \rho D^{*}
\end{aligned}
$$

and find that

$$
\Phi\left(\delta_{H}(x)\right)-\delta_{H}(\Phi(x))=\left(\lambda^{2}-1\right)\left(D[\rho, x] D^{*}-D^{*}[\rho, x] D\right)
$$

which is non-zero, e.g. for $x=\left|e_{1}\right\rangle\left\langle e_{2}\right|$.

Note that $\rho$ is the unique invariant state because the QMS is irreducible because the commutant of $\left\{H, D, D^{*}\right\}$, i.e. the fixed point algebra of the QMS generated by $\mathcal{T}$, is trivial (see [12]).

3.2. Quantum symmetric detailed balance conditions. We conclude this discussion dealing with the 1/2-quantum detailed balance condition, or symmetric quantum detailed balance condition

$$
\mathcal{L}-\mathcal{L}^{\prime}=2 i[K, \cdot]
$$

If $\mathcal{T}$ satisfies this condition, by Theorem 10, there exists a special GKSL representation of $\mathcal{L}^{\prime}$ with $G^{\prime}=G-i(2 K+c)$ and $L_{k}^{\prime}=\sum_{j} u_{k j} L_{j}$ for some unitary matrix $\left(u_{k j}\right)_{k j}$; therefore, by Theorem 11 we get 
1. $G \rho^{1 / 2}=\rho^{1 / 2} G^{*}-(2 i K+i c) \rho^{1 / 2}$ for some $c \in \mathbb{R}$,

2. $\rho^{1 / 2} L_{k}^{*}=\sum_{\ell} u_{k \ell} L_{\ell} \rho^{1 / 2}$, for all $k$, for some unitary matrix $\left(u_{k \ell}\right)_{k \ell}$.

Conversely, these conditions ensure the symmetric quantum detailed balance condition; in fact, we proved in [15] that condition 1 easily follows by 2 and by the invariance of $\rho$. Hence, we obtain:

THEOREM 19. $\mathcal{T}$ satisfies the symmetric quantum detailed balance condition if and only if there exists a special GKSL representation of the generator $\mathcal{L}$ by means of operators $H, L_{k}$ such that $\left\{L_{k}\right\}$ satisfies

$$
\rho^{1 / 2} L_{k}^{*}=\sum_{\ell} u_{k \ell} L_{\ell} \rho^{1 / 2},
$$

for all $k$, for some unitary matrix $\left(u_{m \ell}\right)_{m \ell}$.

This means that the symmetric quantum detailed balance condition depends only on the completely positive part of the generator $\mathcal{L}$. More precisely:

THEOREM 20. Let $\mathcal{L}(a)=G a+\Phi(a)+a G^{*}$ be a special representation of $\mathcal{L}$. The following conditions are equivalent:

1. $\mathcal{T}$ satisfies the quantum symmetric detailed balance condition;

2. $\Phi$ is $1 / 2$-symmetric wrt $\rho$.

A condition on $G$ (with $K=0$ ) is necessary to find $\mathcal{T} 1 / 2$-symmetric.

THEOREM 21. $\mathcal{T}$ is KMS-symmetric if and only if one of the equivalent conditions below holds:

(i) there exists a special GKSL representation of $\mathcal{L}$ by means of operators $G, L_{k}$ with

(1) $G \rho^{1 / 2}=\rho^{1 / 2} G^{*}+i c \rho^{1 / 2}$ for some $c \in \mathbb{R}$,

(2) $\rho^{1 / 2} L_{k}^{*}=\sum_{\ell} u_{k \ell} L_{\ell} \rho^{1 / 2}$, for all $k$, for some unitary matrix $\left(u_{k \ell}\right)_{k \ell}$;

(ii) there exists a special GKSL representation of $\mathcal{L}, \mathcal{L}(a)=G a+\Phi(a)+a G^{*}$ such that

$\left(1^{\prime}\right) G \rho^{1 / 2}=\rho^{1 / 2} G^{*}+i c \rho^{1 / 2}$ for some $c \in \mathbb{R}$,

$\left(2^{\prime}\right) \Phi$ is $1 / 2$-symmetric wrt $\rho$.

REMARK 22. Using the modular theory, we showed in [15] that (1), (2) in item (i) of Theorem 21 can be replaced by the following conditions:

$\left(1^{\prime \prime}\right) H=\sum_{k} H_{k}+c$ for some $c \in \mathbb{R}$, where

$$
H_{k}:=i \int \sigma_{t}\left(\rho^{-1 / 4} L_{k}^{*} L_{k} \rho^{1 / 4}-\rho^{1 / 4} L_{k}^{*} L_{k} \rho^{-1 / 4}\right)\left(e^{2 \pi t}+e^{-2 \pi t}\right)^{-1} d t ;
$$

$\left(2^{\prime \prime}\right) \rho^{1 / 2} L_{k}^{*}=\sum_{\ell} u_{k \ell} L_{\ell} \rho^{1 / 2}$, for all $k$, for some unitary matrix $\left(u_{k \ell}\right)_{k \ell}$.

This is exactly the result proved by Park in Theorem 2.2 of [23].

Acknowledgements. The first author would like to thank the organizers of the $11^{\text {th }}$ Będlewo workshop on Non-Commutative Harmonic Analysis with Applications to Proba- 
bility for the warm hospitality and nice atmosphere. He also acknowledges conversations with R. Hudson and A. Skalski.

\section{References}

[1] L. Accardi and K. Imafuku, Dynamical detailed balance and local KMS condition for non-equilibrium states, Internat. J. Modern Phys. B 18 (2004), 435-467.

[2] L. Accardi and A. Mohari, Time reflected Markov processes, Infinite Dim. Anal. Quantum Probab. Related Topics 2 (1999), 397-426.

[3] S. Albeverio and G. Goswami, A remark on the structure of symmetric quantum dynamical semigroups, Infinite Dim. Anal. Quantum Probab. Related Topics 5 (2002), 571-579.

[4] R. Alicki, On the detailed balance condition for non-Hamiltonian systems, Rep. Math. Phys. 10 (1976), 249-258.

[5] R. Alicki and K. Lendi, Quantum Dynamical Semigroups and Applications, Lect. Notes Phys. 286, Springer-Verlag, 1987.

[6] O. Bratteli and D. W. Robinson, Operator Algebras and Quantum Statistical Mechanics I, Springer-Verlag, Berlin 1979.

[7] O. Bratteli and D. W. Robinson, Unbounded derivations of von Neumann algebras, Ann. Inst. H. Poincaré. 25 (2), (1976), 139-164.

[8] F. Cipriani, Dirichlet Forms and Markovian semigroups on standard forms of von Neumann algebras, J. Funct. Anal. 147 (1997), 259-300.

[9] F. Cipriani, Quantum Potential Theory: Structure and Applications to Physics, Lectures of the "School Quantum Potential Theory: Structure and Applications to Physics". Greifswald 2007.

[10] J. Dereziński and R. Fruboes, Fermi golden rule and open quantum systems, in: S. Attal et al. (eds.), Open Quantum Systems III, Lecture Notes in Mathematics 1882, Springer, 2006, 67-116.

[11] F. Fagnola and R. Quezada, Two-photon absorption and emission process, Infinite Dim. Anal. Quantum Probab. Related Topics 8 (2005), 573-59.

[12] F. Fagnola and R. Rebolledo, Subharmonic projections for a quantum Markov semigroup, J. Math. Phys. 43 (2002), 1074-1082.

[13] F. Fagnola and V. Umanità, Generators of detailed balance quantum Markov semigroups, Infin. Dimens. Anal. Quantum Probab. Relat. Top. 10 (2007), 335-363.

[14] F. Fagnola and V. Umanità, Detailed balance, Time reversal and generators of quantum Markov semigroups, Mathematical Notes 84 (2008), 108-115.

[15] F. Fagnola and V. Umanità, Generators of symmetric quantum Markov semigroups and detailed balance, preprint.

[16] A. Frigerio and V. Gorini, Markov dilations and quantum detailed balance, Comm. Math. Phys. 93 (1984), 517-532.

[17] S. Goldstein and J. M. Lindsay, Beurling-Deny condition for KMS-symmetric dynamical semigroups, C. R. Acad. Sci. Paris 317 (1993), 1053-1057.

[18] S. Goldstein and J. M. Lindsay, KMS symmetric semigroups, Math. Z. 219 (1995), 591-608.

[19] A. Kossakowski, A. Frigerio, V. Gorini and M. Verri, Quantum detailed balance and KMS condition, Comm. Math. Phys. 57 (1977), 97-110. 
[20] W. A. Majewski and R. F. Streater, Detailed balance and quantum dynamical maps, J. Phys. A: Math. Gen. 31 (1998), 7981-7995.

[21] W. A. Majewski, On the relationship between the reversibility of detailed balance conditions, Ann. Inst. Henri Poincaré A 39 (1983), 45-54.

[22] W. A. Majewski, The detailed balance condition in quantum statistical mechanics, J. Math. Phys. 25 (1984), 614-616.

[23] Y. M. Park, Remarks on the structure of Dirichlet forms on standard forms of von Neumann algebras, Infin. Dimens. Anal. Quantum Probab. Relat. Top. 8 (2005), 179-197.

[24] K. R. Parthasarathy, An Introduction to Quantum Stochastic Calculus, Monographs in Mathematics 85, Birkhäuser-Verlag, Basel, 1992. 
